\title{
OBEs in the Blind and Encounters with the Light
}

To the Editor:

As one who suggested more than a decade ago the importance of investigating whether congenitally blind persons have out-of-body experiences (OBEs) with a visual component (Krishnan, 1983), I am glad to note that Kenneth Ring and Sharon Cooper are now collecting such claims and examining them (Ring, 1995; Ring and Cooper, 1997). I hope researchers in this area would give some thought to certain suggestions I subsequently made in hopes of finding a way to identify the underlying mechanism (Krishnan, 1988, 1993).

Ring claimed that accurate out-of-body perceptions by blind persons would mean that there is some conscious aspect of ourselves that can function independently of brain-based consciousness. I'm afraid there is no compelling reason to draw this conclusion. Might not out-of-body sight be an entirely brain-based form of perception? I have pointed out why this possibility cannot be dismissed easily (Krishnan, 1985, 1993).

Clearly, Ring and Cooper favor the view that there are two entirely different orders of reality, or "stuff," commonly referred to as matter and non-matter, or spirit. How reasonable is that? The question can be handled in different ways. The following is one of them. If it is held that the two orders of reality have nothing in common between them, then it is obvious that they cannot interact and we cannot 
know anything about the order to which we do not belong; it does not exist for us. But if it is assumed that they can interact, then it would mean that they have something in common and, therefore, they cannot be treated as two distinct orders of reality. So, then, we have to conclude that there is only one order of reality, and terms like "material" and "nonmaterial" are our descriptions of some of its characteristics as understood though the different techniques employed to study its nature. I hope Ring and Cooper will consider whether monism understood in the above sense might not be the truth.

As for the "encounter with the light" (Ring, 1995, p. 129), the few studies of near-death experiences (NDEs) in India that have been published (Osis and Haraldsson, 1977; Pasricha, 1992, 1993) suggested that the experience is rather rare among Indians. This impression may be due to the smallness of the study samples. Anyway, the Indian experiencers do not seem to have been as much impressed by the light as American subjects were. For example, they do not speak about it as an entity radiating love, or with overwhelming emotions of reverence and adoration.

My collection of about 50 NDE accounts confirms this. I should, however, make it clear that not all my interviewees had the light experience in what would usually be called a life-threatening situation. One of them, who had it during meditation, found himself in an "expanse" of a "very light blue" color of light and enjoyed the experience, although he regarded it as hallucination. (His preceptor had told him to ignore all imagery, with or without form.)

There are also some other differences between the NDEs of Indians and Americans (Pasricha, 1993). Such variations in the experiencers' attitude to light or other elements could be due to differences in belief system, culture, deprivation of parental affection, psychological make-up, critical faculty, and such factors, and point to the need for more cross-cultural studies, as well as caution in considering the ontological significance of all elements in the NDE.

\section{References}

Krishnan, V. (1983). Research request: Out-of-body experience in the congenitally blind. Vital Signs, 3(3), 13.

Krishnan, V. (1985). Near-death experiences: Evidence for survival? Anabiosis: The Journal of Near-Death Studies, 5(1), 21-38. 
Krishnan, V. (1988). OBEs in the blind [Letter]. Journal of Near-Death Studies, 7, 134-139.

Krishnan, V. (1993). The physical basis of out-of-body vision [Letter]. Journal of NearDeath Studies, 11, 257-260.

Osis, K, and Haraldsson, E. (1977). At the hour of death. New York, NY: Avon.

Pasricha, S. (1992). Near-death experiences in South India: A systematic survey in Channapatna. National Institute of Mental Health and Neuro Sciences Journal, 10(2), 111-118.

Pasricha, S. (1993). A systematic survey of near-death experiences in South India. Journal of Scientific Exploration, 7, 161-171.

Ring, K. (1995). [Review of Dying to live: Science and the near-death experience]. Journal of Near-Death Studies, 14, 117-132.

Ring, K., and Cooper, S. (1997). Near-death and out-of-body experiences in the blind: A study of apparent eyeless vision. Journal of Near-Death Studies, 16, 101-147.

V. Krishnan

P. O. Box 1863

Kaloor

Cochin 682017

India 